\title{
Correlation between upper and lower airway inflammations in patients with combined allergic rhinitis and asthma syndrome: A comparison of patients initially presenting with allergic rhinitis and those initially presenting with asthma
}

\author{
SHU XIA ${ }^{1,2^{*}}$, ZHENG ZHU $^{1 *}$, WEI-JIE GUAN ${ }^{1}$, YAN-QING XIE ${ }^{1}$, JIA-YING AN $^{1}$, \\ TAO PENG $^{2}$, RONG-CHANG CHEN ${ }^{1}$ and JIN-PING ZHENG ${ }^{1}$

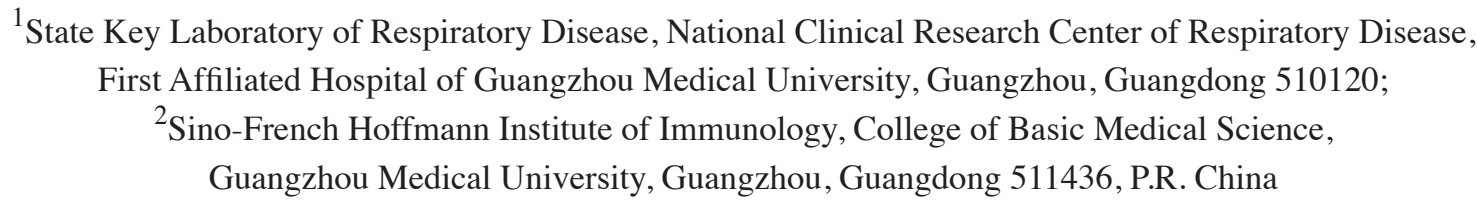

Received March 10, 2017; Accepted September 29, 2017

DOI: $10.3892 / e t m .2017 .5536$

\begin{abstract}
Allergic rhinitis (AR) and asthma often coexist. The terminology combined allergic rhinitis and asthma syndrome (CARAS) was introduced to describe patients with combined AR and asthma. The aim of the present study was to evaluate the correlation between eosinophilic inflammation in the upper and lower airways of patients with CARAS. Stable patients with CARAS initially presenting with AR or asthma were recruited. Healthy subjects and patients with AR alone were recruited as controls. Clinical characteristics, including disease history, lung function, nasal airway inspiratory resistance and upper and lower airway eosinophilic inflammation were evaluated and compared. A total of 73 subjects ( 22 patients with CARAS initially presenting with AR, 15 patients with CARAS initially presenting with asthma, 25 patients with AR alone and 11 healthy subjects) were studied. The nasal symptoms visual analogue scale scores at the week prior to enrollment and nasal airway inspiratory resistances were comparable among the groups. The percentage of predicted forced expiratory volume in $1 \mathrm{sec}$ and percentage of predicted maximal middle expiratory flow in patients with CARAS initially presenting with asthma were significantly lower
\end{abstract}

Correspondence to: Dr Jin-Ping Zheng, State Key Laboratory of Respiratory Disease, National Clinical Research Center of Respiratory Disease, First Affiliated Hospital of Guangzhou Medical University, 151 Yanjiang Road, Guangzhou, Guangdong 510120, P.R. China

E-mail: jpzhenggy@163.com

${ }^{*}$ Contributed equally

Key words: allergic rhinitis, asthma, combined allergic rhinitis and asthma syndrome, eosinophil, airway inflammation, correlation compared with the other three groups $(\mathrm{P}<0.05)$. No significant different in the percentage of eosinophils in the nasal lavage was observed between patients with CARAS and those with AR only; however, it was significantly increased compared with healthy subjects $(\mathrm{P}<0.05)$. The fractional concentration of exhaled nitric oxide and percentage of eosinophils in the sputum were significantly increased in patients with CARAS compared with those in the AR only and healthy subject groups $(\mathrm{P}<0.05)$. The difference in the percentage of eosinophils in the nasal lavage and sputum between patients with CARAS initially presenting with AR and initially presenting with asthma was not significant; however, a positive correlation between the percentage of eosinophils in the upper and lower airways was present in patients with CARAS initially presenting with AR only $(\mathrm{r}=0.526, \mathrm{P}=0.030)$.

\section{Introduction}

Allergic rhinitis (AR) often coexists with asthma. Up to $80 \%$ of patients with asthma are affected by AR and $40 \%$ of AR cases are concomitant with asthma (1-3). It is therefore hypothesized that AR and asthma may represent two manifestations of the same disease (4). The new terminology, combined AR and asthma syndrome (CARAS), was introduced by the world allergy organization to underline the link of the allergic disease between the upper and lower airways (5).

The concept of 'one airway one disease' or united airway disease has been widely accepted $(3,6)$. Compared with AR alone, the combination of upper and lower airway disease is a manifestation of a more severe form of a syndrome affecting the entire airway (5). The inflammatory processes may be the most important link in the cross-talk between the upper and lower airways. Studies have reported that AR impairs the control of asthma by enhancing lower airway inflammation and allergen nasal challenge may aggravate lower airway inflammation and hyper responsiveness $(7,8)$. In addition, bronchial allergen challenge increases systemic and nasal 
mucosal inflammation (9). It has been demonstrated that intranasal corticosteroids improve asthma-specific outcomes, including asthma symptom scores, rescue medication use and morning and evening peak expiratory flow in patients with CARAS (4). Conversely, it has also been reported that nasal allergen exposure has no effects on lower airway inflammation (10).

In a previous study, it was reported that either AR symptoms presented prior to asthma or asthma symptoms presented prior to AR during the study period (2). The association between eosinophilic inflammation in the upper and lower airways may differ in patients with CARAS initially presenting with AR and patients initially presenting with asthma; however, this remains unclear.

The aim of the present study was to evaluate and compare the association between eosinophils in the upper and lower airways of patients with CARAS initially presenting with AR and those initially presenting with asthma.

\section{Patients and methods}

Study design. Following screening, the disease history of patients was recorded using a questionnaire. Nasal lavage, induced sputum, measurement of fractional concentration of exhaled nitric oxide (FeNO), quality of life by Rhinoconjunctivitis Quality of Life Questionnaire (RQLQ) and lung function tests were performed within $24 \mathrm{~h}$. Differential cell counting following nasal lavage and induced sputum was performed. The study protocol was approved by the Ethics Committee of the First Affiliated Hospital of Guangzhou Medical University (Guangzhou, China) and written informed consent was obtained from all participants.

Subjects. A total of 73 subjects were recruited from the Outpatient Department of The First Affiliated Hospital of Guangzhou Medical University (Guangzhou, China) between June 2013 and June 2014. The subjects were divided into the following groups: A CARAS-AR group consisting of patients with CARAS initially presenting with AR $(n=22$; mean age, 31.1; 4 males and 18 females); a CARAS-AS group consisting of patients with CARAS initially presenting with asthma $(n=15$; mean age, 33.6 \pm 11.2 years; 4 males and 11 females); an AR only group consisting of patients with AR only $(n=25$; mean age, $20.5 \pm 2.7$ years; 8 males and 13 females); and a healthy subjects group ( $\mathrm{n}=11$; mean age, 21.1 \pm 3.4 years; 8 males and 3 females). The diagnosis of AR and asthma was made according to the International Guidelines of Allergic Rhinitis and its Impact on Asthma and Global Initiative for Asthma $(6,11)$. Patients with AR had recurrent nasal symptoms, including sneezing, nasal discharge, nasal blockage or itching in the preceding year but no obvious allergen exposure and nasal symptom exacerbation for at least one week prior to the study. Patients with CARAS were stable with no lower airway symptoms, including coughing, breathlessness, chest tightness or wheezing for at least 1 month prior to recruitment, as assessed by Asthma Control Test questionnaire (11). Patients with AR only and patients with CARAS also had positive results from a skin prick test with a panel of aeroallergens, including Dermatophagoides pteronyssinus, Dermatophagoides farina, dogs, cats, Alternaria alternata, Blattella germanica, mixed tree pollen and mixed grass. These patients exhibited appropriate responses to the positive histamine and negative diluent controls (ALK-Abello, Hørsholm, Denmark). Patients who had undergone immunotherapy, been treated with oral corticosteroids within 3 months, had acute upper airway infection within 4 weeks, had any other respiratory disease (e.g. bronchiectasis, chronic obstructive pulmonary disease), were pregnant or lactating, or were current smokers were excluded. Antihistamines, leukotrienes receptor antagonists and intranasal or inhaled corticosteroids were withheld for at least 2 weeks prior to the study. A group of non-atopic healthy subjects without acute airway infection within 4 weeks prior to the study were enrolled as controls.

Measurement of FeNO. FeNO was measured using a chemiluminescence analyzer (NIOX MINO, Aerocrine, Solna, Sweden) according to the international guidelines (12). Patients performed the measurement by inhaling through the device from the functional residual capacity to the total lung capacity, followed by exhaling into the device for $10 \mathrm{sec}$ with a flow rate of $50 \mathrm{ml} / \mathrm{s}$. No more than 8 attempts were allowed for each subject in order to obtain a valid result.

Lung function test. Lung function tests were performed using a spirometer (MasterScreen Diffusion; CareFusion; BD Biosciences, Franklin Lakes, NJ, USA) according to the recommendations of the American Thoracic Society/European Respiratory Society (13). Between 3 and 8 maneuvers were performed with a variation between the best 2 maneuvers of $<5 \%$ or $150 \mathrm{ml}$ in forced vital capacity (FVC) and forced expiratory volume in $1 \mathrm{sec}\left(\mathrm{FEV}_{1}\right)$. The maximal values of the $\mathrm{FVC}$ and $\mathrm{FEV}_{1}$ were reported.

Nasal airway resistance (NAR) measurement. NAR was measured with an active anterior rhinomanometry using a rhinomanometer (MasterScreen Diffusion; CareFusion; BD Biosciences) according to international guidelines (14). Between 3 and 5 rhinomanometric maneuvers were performed in each nostril at a pressure of $150 \mathrm{~Pa}$.

Nasal lavage. Nasal lavage was performed bilaterally using a syringe with a sponge adapter with the patient's head in a forward position. Sterile normal saline solution $(10 \mathrm{ml})$ was infused into the nostrils and lavage fluid was obtained for subsequent experimentation. Lavage fluids were immediately centrifuged for $10 \mathrm{~min}$ at $2,000 \mathrm{x} \mathrm{g}$ at $4^{\circ} \mathrm{C}$ and cytospin slides were air-dried, fixed for $10 \mathrm{~min}$ in $4 \%$ formaldehyde at $24^{\circ} \mathrm{C}$, and stained with hematoxylin and eosin at $24^{\circ} \mathrm{C}$ for $1 \mathrm{~min}$. The results were expressed as percentages of neutrophils, eosinophils, macrophages and lymphocytes, which were measured via light microscopy at x200 magnification (50i; Nikon Corporation, Tokyo, Japan).

Induced sputum. Sputum was induced with an aerosol of hypertonic saline (3\%) for 10-20 min. The sputum plug was collected and treated with dithioerythritol, vortexed for $10 \mathrm{sec}$ at $2,000 \mathrm{x} \mathrm{g}$ and $24^{\circ} \mathrm{C}$, and incubated at $37^{\circ} \mathrm{C}$ in a water bath for $15 \mathrm{~min}$. The suspension was filtered through a $48 \mu \mathrm{m}$ nylon filter and subsequently centrifuged for $10 \mathrm{~min}$ at $3,000 \mathrm{x}$ g and $4^{\circ} \mathrm{C}$. Samples were then air-dried, fixed for $10 \mathrm{~min}$ in $4 \%$ formaldehyde at $24^{\circ} \mathrm{C}$, 
Table I. Demographic characteristics.

\begin{tabular}{|c|c|c|c|c|c|}
\hline Characteristics & $\begin{array}{c}\text { Healthy } \\
\text { subjects }(\mathrm{n}=11)\end{array}$ & $\begin{array}{c}\text { AR } \\
\text { only }(n=25)\end{array}$ & $\begin{array}{c}\text { CARAS initially } \\
\text { presenting with AR }(n=22)\end{array}$ & $\begin{array}{c}\text { CARAS initially } \\
\text { presenting with asthma }(n=15)\end{array}$ & P-value \\
\hline Age (years) & $21.1 \pm 3.4$ & $20.5 \pm 2.7$ & $31.1 \pm 8.8^{\mathrm{a}, \mathrm{b}}$ & $33.6 \pm 11.2^{\mathrm{a}, \mathrm{b}}$ & $<0.05$ \\
\hline Height $(\mathrm{cm})$ & $165.1 \pm 9.0$ & $165.1 \pm 7.9$ & $159.6 \pm 6.2$ & $159.5 \pm 8.3$ & $>0.05$ \\
\hline Weight (kg) & $57.0 \pm 7.8$ & $53.5 \pm 7.1$ & $52.2 \pm 9.6$ & $58.7 \pm 10.6$ & $>0.05$ \\
\hline Gender $(\mathrm{M} / \mathrm{F})$ & $8 / 3$ & $8 / 3$ & $4 / 18^{\mathrm{a}, \mathrm{b}}$ & $4 / 11^{\mathrm{a}, \mathrm{b}}$ & $<0.05$ \\
\hline \multicolumn{6}{|l|}{ Disease history (y) } \\
\hline $\mathrm{AR}$ & - & $5.4 \pm 2.6$ & $13.1 \pm 8.9^{b}$ & $11.0 \pm 10.8^{\mathrm{b}}$ & $<0.05$ \\
\hline Asthma & - & - & $3.46 \pm 4.4$ & $18.1 \pm 14.1$ & $>0.05$ \\
\hline \multicolumn{6}{|l|}{ Allergens (n) } \\
\hline $\operatorname{Der} p$ & - & 15 & 16 & 14 & $>0.05$ \\
\hline $\operatorname{Derf}$ & - & 15 & 17 & 12 & $>0.05$ \\
\hline Tropical mite & - & 12 & 14 & 6 & $>0.05$ \\
\hline Dogs & - & 2 & 7 & 4 & $>0.05$ \\
\hline Cats & - & 5 & 5 & 3 & $>0.05$ \\
\hline Alternaria alternata & - & 2 & 1 & 0 & $>0.05$ \\
\hline Blattella germanica & - & 3 & 9 & 7 & $>0.05$ \\
\hline Mixed tree pollen & - & 0 & 0 & 0 & $>0.05$ \\
\hline Mixed grass & - & 2 & 0 & 1 & $>0.05$ \\
\hline $\mathrm{FEV}_{1} \%$ pred & $92.0 \pm 9.4$ & $88.8 \pm 8.5$ & $84.0 \pm 7.4^{\mathrm{a}}$ & $76.9 \pm 8.4^{\mathrm{a}, \mathrm{b}}$ & $<0.05$ \\
\hline MMEF\%pred & $75.8 \pm 32.3$ & $80.6 \pm 19.1$ & $62.4 \pm 8.5$ & $44.2 \pm 16.6^{\mathrm{b}}$ & $<0.05$ \\
\hline NAR & $0.19 \pm 0.07$ & $0.24 \pm 0.13$ & $0.23 \pm 0.06$ & $0.21 \pm 0.05$ & $>0.05$ \\
\hline VAS & - & $3.2 \pm 1.5$ & $3.4 \pm 2.5$ & $4.3 \pm 2.9$ & $>0.05$ \\
\hline
\end{tabular}

Data are expressed as the mean \pm standard deviation. ${ }^{\mathrm{a}} \mathrm{P}<0.05$ vs. healthy subjects. ${ }^{\mathrm{b}} \mathrm{P}<0.05$ vs. AR only. Der $p$, Dermatophagoides pteronyssinus; Der f, Dermatophagoides farina; $\mathrm{FEV}_{1} \%$ pred, percentage of predicted forced expiratory volume in 1 sec; MMEF\% pred, percentage of predicted maximal middle expiratory flow; NAR, nasal airway inspiratory resistance; VAS, visual analogue scale; AR, allergic rhinitis; CARAS, combined allergic rhinitis and asthma syndrome.

and stained with hematoxylin for $10 \mathrm{sec}$ and eosin for $1 \mathrm{~min}$ at $24^{\circ} \mathrm{C}$. A total of 400 nonsquamous cells were counted. Samples with cell viability $>70 \%$ and squamous cells $<20 \%$ were considered adequate. The results were expressed as percentages of neutrophils, eosinophils, macrophages and lymphocytes, which were measured via light microscopy at x200 magnification (50i; Nikon Corporation).

Statistical analysis. Statistical analysis was performed using SPSS 16.0 (SPSS, Inc., Chicago, IL, USA). Data with a normal distribution were expressed as the mean \pm standard deviation; otherwise the median (interquartile range) was used. One-way analysis of variance with Tukey's post hoc test was performed for comparison of multiple groups. Pearson's correlation analysis was performed to evaluate the relationship between eosinophils in the upper and lower airways. $\mathrm{P}<0.05$ was determined to indicate a statistically significant difference.

\section{Results}

Subjects. A total of 73 subjects (22 patients with CARAS initially presenting with AR, 15 patients with CARAS initially presenting with asthma, 25 patients with AR only and 11 healthy subjects) were enrolled in the present study (Table I).
CARAS Patients had significantly longer history of AR compared with patients with AR only, and CARAS patients initially presenting with asthma had significantly lower $\mathrm{FEV}_{1}$ (FEV ${ }_{1} \%$ pred) and lower maximal middle expiratory flow (MMEF\% pred) compared with patients with AR only (both $\mathrm{P}<0.05$; Table I). No significant difference in NARs score was observed between groups.

Nasal lavage and induced sputum. No significant difference in the percentage of eosinophils in nasal lavage was observed between the AR only and CARAS-AR and CARAS-AS groups; however, this percentage was significantly increased in all groups compared with the healthy subjects $(\mathrm{P}<0.05$; Table II). The percentage of eosinophils in induced sputum was significantly higher in the CARAS-AR and CARAS-AS groups compared with the AR only and healthy subject groups $(\mathrm{P}<0.05$; Table II). However, no significant difference was in the percentage of eosinophils in induced sputum was observed between the CARAS-AR and CARAS-AS groups (Table II). The percentage of eosinophils in the nasal lavage was positively correlated with that in induced sputum in the CARAS-AR group only $(r=0.526, P=0.030$; Fig. 1 and Table III). No significant correlation was observed between the percentage of eosinophils in the upper and lower airways in any other groups (Table III). 
Table II. Eosinophils in nasal lavage and induced sputum.

\begin{tabular}{|c|c|c|c|c|c|}
\hline Sample & $\begin{array}{c}\text { Healthy } \\
\text { subjects }(n=11)\end{array}$ & $\begin{array}{c}\text { AR } \\
\text { only }(n=25)\end{array}$ & $\begin{array}{c}\text { CARAS initially } \\
\text { presenting with AR }(n=22)\end{array}$ & $\begin{array}{c}\text { CARAS initially } \\
\text { presenting with asthma }(n=15)\end{array}$ & P-value \\
\hline \multicolumn{6}{|l|}{ Nasal lavage } \\
\hline Eosinophils (\%) & $4.32 \pm 6.35$ & $37.82 \pm 29.13^{\mathrm{a}}$ & $46.73 \pm 25.19^{\mathrm{a}}$ & $41.02 \pm 27.85^{\mathrm{a}}$ & $<0.05$ \\
\hline Neutrophils (\%) & $81.60 \pm 29.88$ & $49.88 \pm 31.14^{\mathrm{a}}$ & $50.41 \pm 26.10^{\mathrm{a}}$ & $56.98 \pm 26.54^{\mathrm{a}}$ & $<0.05$ \\
\hline \multicolumn{6}{|l|}{ Induced sputum } \\
\hline Eosinophils (\%) & $0.20 \pm 0.27$ & $4.59 \pm 4.21$ & $17.34 \pm 13.49^{\mathrm{a}, \mathrm{b}}$ & $17.19 \pm 12.97^{\mathrm{a}, \mathrm{b}}$ & $<0.05$ \\
\hline Neutrophils (\%) & $58.90 \pm 21.47$ & $68.11 \pm 24.58$ & $58.32 \pm 27.12$ & $59.93 \pm 21.56$ & $>0.05$ \\
\hline
\end{tabular}

Data are expressed as the mean \pm standard deviation. ${ }^{a} \mathrm{P}<0.05$ vs. healthy subjects. ${ }^{b} \mathrm{P}<0.05 \mathrm{vs}$. AR only. AR, allergic rhinitis; CARAS, combined allergic rhinitis and asthma syndrome.

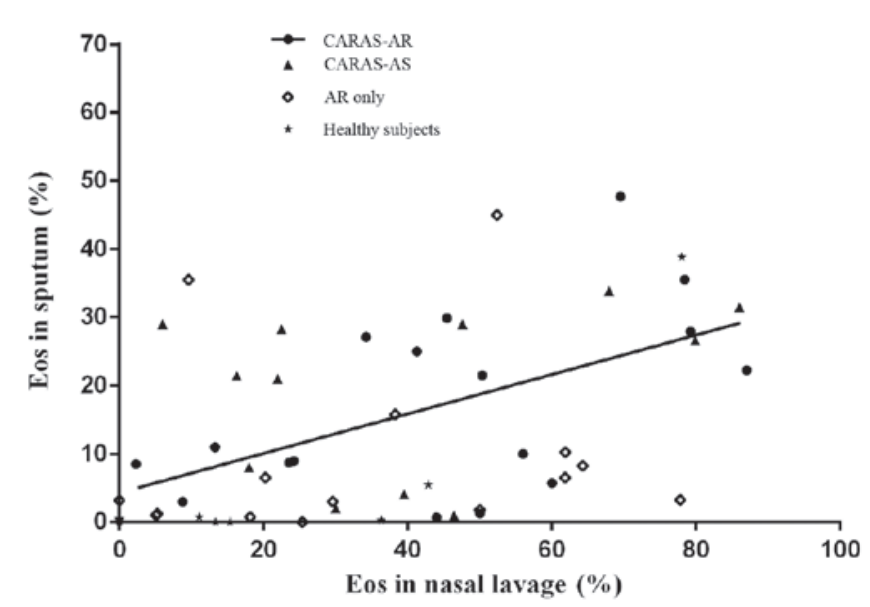

Figure 1. Correlations of eosinophils in sputum and nasal lavage. A positive correlation between the upper and lower airway eosinophils was present in patients with CARAS initially presenting with AR only $(r=0.526$, $\mathrm{P}=0.030$ ). CARAS-AR, patients with CARAS initially presenting with AR; CARAS-AS, patients with CARAS initially presenting with asthma; AR, allergic rhinitis; Eos, eosinophils; CARAS, combined allergic rhinitis and asthma syndrome.

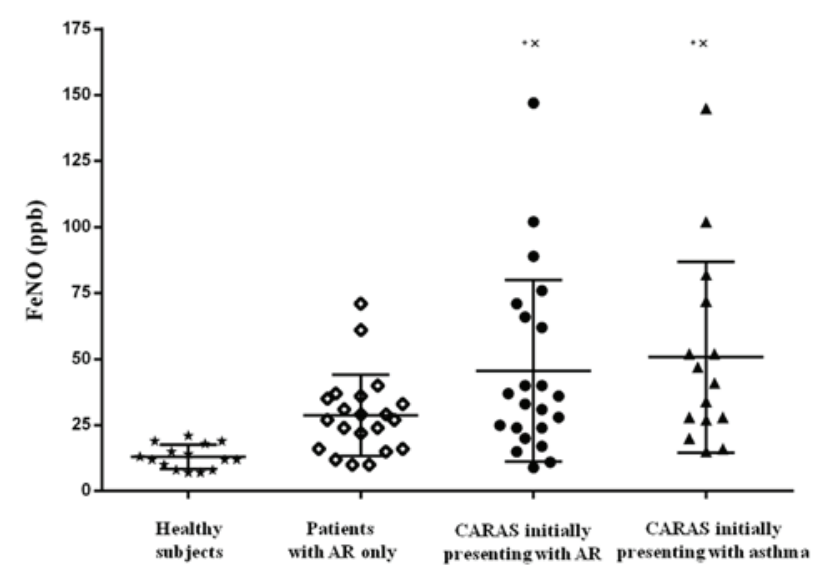

Figure 2. Distributions of FeNO in different groups. There was significantly increased FeNO in patients with CARAS initially presenting with AR (34.5 (44) $\mathrm{ppb}$ ) and initially presenting with asthma (41.0 (45) ppb) compared with those in the AR only (27.0 (20) ppb) and healthy subject (12.5 (10) ppb) groups. Data are expressed as the median and interquartile range. ${ }^{*} \mathrm{P}<0.05$ vs. healthy subject group. ${ }^{x} \mathrm{P}<0.05$ vs. AR only group. AR, allergic rhinitis; Eos, eosinophils; CARAS, combined allergic rhinitis and asthma syndrome; FeNO, fractional exhaled nitric oxide.

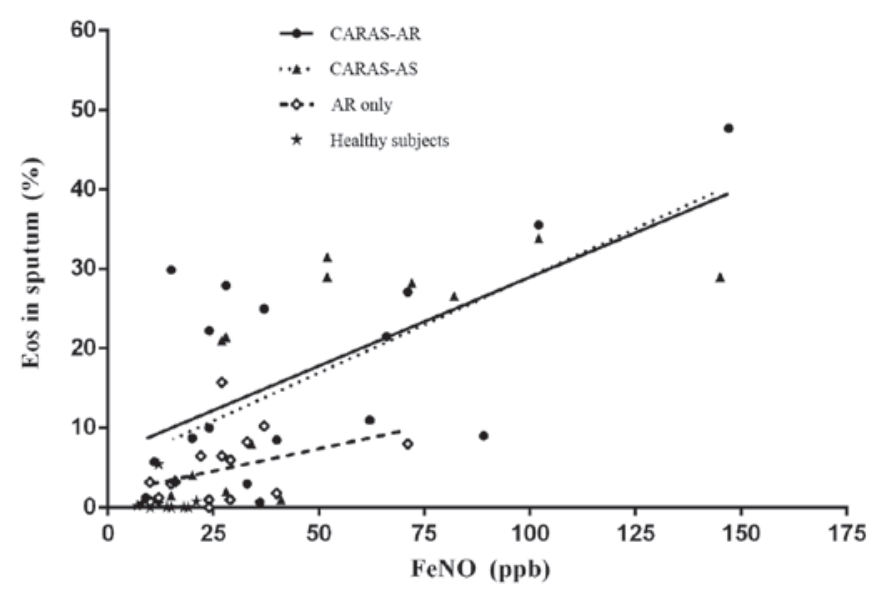

Figure 3. Correlations of FeNO with eosinophils in sputum. The FeNO and eosinophils in sputum were positively correlated in patients with CARAS initially presenting with AR, patients with CARAS initially presenting with asthma and patients with AR only $(\mathrm{r}=0.62,0.70$, and 0.76 , respectively; all $\mathrm{P}<0.05)$. CARAS-AR, patients with CARAS initially presenting with AR; CARAS-AS, patients with CARAS initially presenting with asthma; AR, allergic rhinitis; Eos, eosinophils; CARAS, combined allergic rhinitis and asthma syndrome; FeNO, fractional exhaled nitric oxide.

FeNO. The level of FeNO in the CARAS-AR and CARAS-AS groups was significantly increased compared with the AR only and healthy subject groups $(\mathrm{P}<0.05$; Fig. 2$)$, whereas the difference between the CARAS-AS and CARAS-AR groups was not significant (Fig. 2). FeNO was positively correlated with the percentage of eosinophils in sputum in the AR only, CARAS-AR and CARAS-AS groups $(r=0.616, P=0.008$ and $r=0.701, P=0.005$, respectively; Fig. 3).

Quality of life. RQLQ scores were reported in the CARAS-AR and AR only groups compared with those in the CARAS-AS group, with the highest total scores reported in the CARAS-AR group, especially in terms of sleep problems and emotional function (Table IV).

\section{Discussion}

In the present study, no significant difference was observed in the percentage of eosinophils in nasal lavage in the AR only, CARAS-AR and CARAS-AS groups. A previous study reported 
Table III. Pearson's correlation of inflammation in the upper and lower airway.

\begin{tabular}{|c|c|c|c|}
\hline \multirow[b]{2}{*}{ Nasal lavage } & \multicolumn{2}{|c|}{ Induce sputum } & \multirow[b]{2}{*}{$\mathrm{FeNO}(\mathrm{ppb})$} \\
\hline & $\operatorname{Eos}(\%)$ & $\mathrm{Nu}(\%)$ & \\
\hline \multicolumn{4}{|l|}{ Healthy subjects } \\
\hline Eosinophils (\%) & $0.239(0.479)$ & $-0.546(0.082)$ & $-0.221(0.467)$ \\
\hline Neutrophils (\%) & $-0.033(0.924)$ & $0.673^{\mathrm{a}}(0.023)$ & $-0.087(0.777)$ \\
\hline FeNO (ppb) & $-0.111(0.732)$ & $0.057(0.861)$ & \\
\hline \multicolumn{4}{|l|}{ AR only } \\
\hline Eosinophils (\%) & $0.405(0.135)$ & $-0.232(0.406)$ & $0.207(0.411)$ \\
\hline Neutrophils (\%) & $-0.296(0.284)$ & $-0.083(0.770)$ & $-0.353(0.150)$ \\
\hline FeNO (ppb) & $0.764^{\mathrm{b}}(0.001)$ & $-0.461(0.072)$ & \\
\hline \multicolumn{4}{|c|}{ CARAS initially presenting with AR } \\
\hline Eosinophils (\%) & $0.526^{\mathrm{a}}(0.030)$ & $-0.138(0.597)$ & $0.075(0.761)$ \\
\hline Neutrophils (\%) & $-0.535^{\mathrm{a}}(0.027)$ & $0.127(0.627)$ & $-0.135(0.583)$ \\
\hline FeNO (ppb) & $0.619^{\mathrm{b}}(0.008)$ & $-0.379(0.134)$ & \\
\hline \multicolumn{4}{|c|}{ CARAS initially presenting with asthma } \\
\hline Eosinophils (\%) & $0.315(0.318)$ & $-0.347(0.269)$ & $0.392(0.208)$ \\
\hline Neutrophils (\%) & $-0.329(0.297)$ & $0.351(0.263)$ & $-0.379(0.225)$ \\
\hline FeNO (ppb) & $0.701^{\mathrm{b}}(0.005)$ & $-0.789^{b}(0.001)$ & \\
\hline
\end{tabular}

Data are expressed as the mean \pm standard deviation. ${ }^{\mathrm{a}} \mathrm{P}<0.05 .{ }^{\mathrm{b}} \mathrm{P}<0.01$. Eos, eosinophil; Nu, neutrophil; AR, allergic rhinitis; CARAS, combined allergic rhinitis and asthma syndrome.

Table IV. The RQLQ scores in different groups.

\begin{tabular}{|c|c|c|c|c|}
\hline $\begin{array}{l}\text { Domains } \\
\text { of RQLQ }\end{array}$ & $\begin{array}{c}\text { AR } \\
\text { only }(n=25)\end{array}$ & $\begin{array}{c}\text { CARAS initially } \\
\text { presenting with AR }(n=22)\end{array}$ & $\begin{array}{c}\text { CARAS initially } \\
\text { presenting with asthma }(n=15)\end{array}$ & P-values \\
\hline Activities & $6.5 \pm 3.6$ & $6.2 \pm 3.3$ & $3.4 \pm 4.0$ & $>0.05$ \\
\hline Sleep problems & $3.6 \pm 3.6$ & $6.5 \pm 4.3^{\mathrm{a}}$ & $4.0 \pm 2.7$ & $<0.05$ \\
\hline Non-nose/eye symptoms & $11.1 \pm 7.2$ & $14.5 \pm 9.1$ & $9.6 \pm 6.4$ & $>0.05$ \\
\hline Practical problems & $9.1 \pm 4.2$ & $8.9 \pm 5.2$ & $6.0 \pm 4.3$ & $>0.05$ \\
\hline Nasal problems & $10.8 \pm 5.1$ & $12.5 \pm 5.8$ & $7.7 \pm 4.8$ & $>0.05$ \\
\hline Ocular problems & $5.1 \pm 3.5$ & $7.3 \pm 5.6$ & $5.4 \pm 5.0$ & $>0.05$ \\
\hline Emotion function & $5.4 \pm 3.4$ & $9.1 \pm 6.7^{\mathrm{a}, \mathrm{c}}$ & $3.4 \pm 3.7^{\mathrm{b}}$ & $<0.05$ \\
\hline Total & $51.6 \pm 20.8$ & $64.9 \pm 32.1^{\mathrm{c}}$ & $39.6 \pm 26.8^{b}$ & $<0.05$ \\
\hline
\end{tabular}

Data are expressed as the mean \pm standard deviation. ${ }^{a} \mathrm{P}<0.05$ vs. AR only. ${ }^{\mathrm{b}} \mathrm{P}<0.05$ vs. CARAS-AR. ${ }^{\mathrm{c}} \mathrm{P}<0.05$ vs. CARAS-AS. AR, allergic rhinitis; CARAS, combined allergic rhinitis and asthma syndrome; RQLQ, rhinoconjunctivitis quality of life questionnaire.

that, compared with AR alone, patients with CARAS have stronger responses to cold air nasal challenge with an increased release of inflammation mediators in nasal secretions and a higher degree of functional abnormality of the entire airway (15). However, in the present study, under normal conditions, lower lung function parameters ( $\mathrm{FEV}_{1} \%$ pred and MMEF\%pred) were present in patients with CARAS compared with AR only and healthy subjects, whereas the percentage of eosinophils in nasal lavage was similar to patients with CARAS and AR only.

The number of eosinophils in the sputum was significantly higher in the CARAS-AS and CARAS-AR groups compared with the AR only and healthy subject groups. Although there eosinophilic inflammation is present in the lower airways of patients with AR only, it may be decreased in patients with AR only compared with patients of CARAS (16). In some patients, AR may develop into asthma under persistent exposure to allergens (2). No significant differences were observed in the percentage of eosinophils in the upper and lower airways in the CARAS-AR and CARAS-AS groups, which suggests that patients with CARAS initially presenting with AR and patients with those initially presenting with asthma may have similar inflammatory mechanisms in the upper and lower airways. This suggests that AR and asthma may represent two manifestations of the same disease (4). 
It has previously been demonstrated that there is an association between nasal and bronchial responsiveness to nonspecific agents in asthmatic patients with AR (17). In addition, airway hyper-responsiveness is positively correlated with eosinophilic inflammation in patients with CARAS $(18,19)$. In the present study, under natural environment allergen exposure, a positive correlation between the percentage of eosinophils in the upper and lower airways was present in the CARAS-AR group only. Previous studies have reported that allergen nasal challenge aggravates lower airway eosinophils (8) and segmental bronchial allergen challenge may induce an increase in eosinophils in the nasal mucosa and bronchia, as well as peripheral blood (9). Thus, the upper and lower airways may have a bidirectional impact on each other.

Several proposed mechanisms may account for the interaction between the upper and lower airways in AR that develops into asthma, including nasal-bronchial reflex, mouth breathing caused by severe nasal obstruction, aspiration of nasal contents and common mucosal inflammatory processes (4). The interactions are not limited to local inflammation, as nasal allergen and bronchial allergen challenge may also induce an increase in systemic inflammation. Interactions between the upper and lower airways primarily occur through systemic inflammation $(8,9)$, especially in CARAS cases in which asthma developed first. The majority of allergen airway challenge studies are primarily focused on the upper or lower airways in AR with or without asthma (8-10). Results have demonstrated the impact of AR on asthma and the benefit of treating $\operatorname{AR}$ in asthma $(17,20)$. The different impacts of allergen nasal and bronchial challenge in local airways and systemic inflammation in CARAS between patients initially presenting with AR and initially presenting with asthma remains unknown. Previous studies have demonstrated that intranasal corticosteroids may improve asthma symptoms in patients with CARAS $(21,22)$. However, the results remain inconsistent $(17,20-22)$. In a long-term observation and follow-up study among patients with asthma and AR, improvements in AR were associated with the resolution of asthma symptoms, whereas worsening of AR was associated with persisting asthma symptoms. Asthma improvement was also associated with the resolution of AR symptoms, whereas worsening of asthma was associated with persisting AR symptoms (20). However, further comparisons of CARAS in patients initially presenting with AR and those initially presenting with asthma is required.

Levels of FeNO may be affected by AR, however they primarily correlate with eosinophils in the lower airways of patients with AR and asthma $(10,18)$. In the present study, the level of FeNO was positively correlated with eosinophils in the lower airways of the AR only, CARAS-AS and CARAS-AR groups. FeNO may therefore be an ideal non-invasive measurement for diagnosing asthma or detecting lower airway involvement in AR (16).

RQLQ has been widely used alongside objective measurements for the assessment and management of AR (23). Higher RQLQ scores have been reported in the CARAS-AR and AR only groups compared with the CARAS-AS group, especially in terms of sleep problems and emotional function. Several factors may account for these differences. Patients with
CARAS initially presenting with AR have a longer AR history; these patients may have more complaints and focus mainly on the symptoms of asthma and overlook AR. In addition, the severity of AR symptoms may affect the RQLQ scores, although in the previous week the visual analogue scale scores were comparable between groups.

The present study is not without limitations. It is an observation study without intervention and follow-up; patients with CARAS for whom AR and asthma developed at the same time were not included and compared in this study. Furthermore, the number of participants in each group was small.

In conclusion, patients with CARAS initially presenting with AR or those initially presenting with asthma have similar nasal and bronchial eosinophilic inflammation, supporting the hypothesis that AR and asthma share common allergic inflammation mechanisms. However, correlations of the eosinophils between the upper and lower airways were found only in patients with CARAS initially presenting with AR. Correlations between the upper and lower airways in patients with CARAS initially presenting with AR and those initially presenting with asthma require further validation using a larger sample cohort. The results of the present study indicate that treatment of AR may benefit the lower airways in patients with AR only or CARAS in clinical practice.

\section{Acknowledgements}

This study was supported by The National Key Scientific \& Technology Support Program (grant no. 2015BAI12B10).

\section{References}

1. Leynaert B, Neukirch F, Demoly P and Bousquet J: Epidemiologic evidence for asthma and rhinitis comorbidity. J Allergy Clin Immunol 106 (5 Suppl): S201-S205, 2000.

2. Greisner WA III, Settipane RJ and Settipane GA: Co-existence of asthma and allergic rhinitis: A 23-year follow-up study of college students. Allergy Asthma Proc 19: 185-188, 1998.

3. Grossman J: One airway, one disease. Chest 111 (2 Suppl): 11S-16S, 1997.

4. Taramarcaz P and Gibson PG: The effectiveness of intranasal corticosteroids in combined allergic rhinitis and asthma syndrome. Clin Exp Allergy 34: 1883-1889, 2004.

5. World Allergy Organisation. Combined allergic rhinitis and asthma syndrome.http://www.worldallergy. org/professional/allergy_update/caras/airwayssynopsis.shtml.

6. Bousquet J, Khaltaev N, Cruz AA, Denburg J, Fokkens WJ, Togias A, Zuberbier T, Baena-Cagnani CE, Canonica GW, van Weel C, et al: Allergic rhinitis and its impact on asthma (ARIA) 2008 update (in collaboration with the world health organization, GA(2)LEN and AllerGen). Allergy 63 (Suppl 86): S8-S160, 2008.

7. Oka A, Matsunaga K, Kamei T, Sakamoto Y, Hirano T, Hayata A, Akamatsu K, Kikuchi T, Hiramatsu M, Ichikawa T, et al: Ongoing allergic rhinitis impairs asthma control by enhancing the lower airway inflammation. J Allergy Clin Immunol Pract 2: 172-178, 2014.

8. Inal A, Kendirli SG, Yilmaz M, Altintas DU, Karakoc GB and Erdogan S: Indices of lower airway inflammation in children monosensitized to house dust mite after nasal allergen challenge. Allergy 63: 1345-1351, 2008.

9. Braunstahl GJ, Kleinjan A, Overbeek SE, Prins JB, Hoogsteden HC and Fokkens WJ: Segmental bronchial provocation induces nasal inflammation in allergic rhinitis patients. Am J Respir Crit Care Med 161: 2051-2057, 2000.

10. Bergmann-Hug K, Wirth R, Henseler M, Helbling A, Pichler WJ and Schnyder B: Effect of natural seasonal pollen exposure and repeated nasal allergen provocation on elevation of exhaled nitric oxde. Allergy 64: 1629-1634, 2009. 
11. From the global strategy for asthma management and prevention, global initiative for asthma (GINA) 2015. Available from: http://www.ginasthma.org/.

12. American Thoracic Society; European Respiratory Society: ATS/ERS recommendations for standardized procedures for the online and offline measurement of exhaled lower respiratory nitric oxide and nasal nitric oxide, 2005. Am J Respir Crit Care Med 171: 912-930, 2005.

13. Miller MR, Hankinson J, Brusasco V, Burgos F, Casaburi R, Coates A, Crapo R, Enright P, van der Grinten CP, Gustafsson P, et al: Standardization of spirometry. Eur Respir J 26: 319-338, 2005.

14. Clement PA and Gordts F: Standardisation Committee on Objective Assessment of the Nasal Airway, IRS, and ERS Consensus report on acoustic rhinometry and rhinomanometry. Rhinology 43: 169-179, 2005.

15. Hanes LS, Issa E, Proud D and Togias A: Stronger nasal responsiveness to cold air in individuals with rhinitis and asthma, compared with rhinitis alone. Clin Exp Allergy 36: 26-31, 2006

16. Zhu Z, Xie Y, Guan W, Gao Y, Xia S, Zhong N and Zheng J: FeNO for detecting lower airway involvement in patients with allergic rhinitis. Exp Ther Med 12: 2336-2340, 2016.
17. Koh YI and Choi IS: Relationship between nasal and bronchial responsiveness in perennial allergic rhinitic patients with asthma. Int Arch Allergy Immunol 129: 341-347, 2002.

18. de Graaf-in t Veld C, Garrelds IM, Koenders S and Gerth van Wijk R: Relationship between nasal hyperreactivity, mediators and eosinophils in patients with perennial allergic rhinitis and controls. Clin Exp Allergy 26: 903-908, 1996.

19. Brannan JD and Lougheed MD: Airway hyperresponsiveness in asthma: Mechanisms, clinical significance, and treatment. Front Physiol 3: 460, 2012.

20. Greisner WA III, Settipane RJ and Settipane GA: The course of asthma parallels that of allergic rhinitis: A 23-year follow-up study of college students. Allergy Asthma Proc 21: 371-375, 2000.

21. Mener DJ and Lin SY: Improvement and prevention of asthma with concomitant treatment of allergic rhinitis and allergen-specific therapy. Int Forum Allergy Rhinol 5 (Suppl 1): S45-S50, 2015.

22. Kessel A: The impact of intranasal corticosteroids on lung function in children with allergic rhinitis. Pediatr Pulmonol 49: 932-937, 2014.

23. Juniper EF, Guyatt GH, Willan A and Griffith LE: Determining a minimal important change in a disease-specific quality of life questionnaire. J Clin Epidemiol 47: 81-87, 1994. 